\title{
A comparison of monoaxial pedicle screw versus polyaxial pedicle screw in short-segment posterior fixation for the treatment of thoracolumbar fractured vertebra
}

\author{
Wenye Yao ${ }^{1 \#}$, Tonghua Zhou ${ }^{1 \#}$, Kai Huang ${ }^{2 \#}$, Min Dai ${ }^{1}$, Fengbo Mo ${ }^{1}$, Jing Xu ${ }^{1}$, Zhiyou Cao ${ }^{1}$, Qi Lai ${ }^{1}$, \\ Banglin Xie ${ }^{1}$, Runsheng Guo ${ }^{1}$, Bin Zhang ${ }^{1}$ \\ ${ }^{1}$ Department of Orthopaedics, The First Affiliated Hospital of Nanchang University, Nanchang, China; ${ }^{2}$ Department of Orthopedics, Zhabei Central \\ Hospital of Jing'an District, Shanghai, China \\ Contributions: (I) Conception and design: R Guo, B Zhang; (II) Administrative support: M Dai; (III) Provision of study materials or patients: W Yao, \\ T Zhou; (IV) Collection and assembly of data: T Zhou, F Mo, Q Lai; (V) Data analysis and interpretation: K Huang, W Yao, B Xie, Z Cao; (VI) \\ Manuscript writing: All authors; (VII) Final approval of manuscript: All authors. \\ \#These authors contributed equally to this work and are first co-authors. \\ Correspondence to: Bin Zhang, MD; Runsheng Guo. Department of Orthopaedics, The First Affiliated Hospital of Nanchang University, 17 Yongwai \\ Rd., Nanchang 330006, China. Email: 13970823907@163.com; grs330006@126.com.
}

Background: Posterior pedicle screw fixation had been applied to maintain spinal stability and avoid further nerve damage in thoracolumbar fracture. This study aimed to evaluate the efficacy of short-segment posterior fixation with monoaxial pedicle screws versus polyaxial pedicle screws in treating thoracolumbar fracture.

Methods: A total of 75 patients with thoracolumbar fracture who underwent short-segment posterior fixation with monoaxial pedicle screw (group A) or polyaxial pedicle screw (group B) were retrospectively enrolled. The patient demographic and radiological data were analyzed between the two groups.

Results: A total of 63 patients with an average age of $44.7 \pm 11.5$ years were finally recruited in this study. There were no significant differences in age, gender, fracture level, thoracolumbar injury classification and severity scale (TLISS) score, American Spinal Injury Association (ASIA) score, Arbeitsgemeinschaft für Osteosynthesefragen (AO) classification, and hospital stay between the two groups $(\mathrm{P}>0.05)$. At the last follow-up, the prevertebral height ratio and normal-to-injured vertebral height ratio were significantly decreased in group A compared to group $\mathrm{B}(\mathrm{P}=0.027$ and $\mathrm{P}=0.007$, respectively).

Conclusions: Short-segment posterior fixation with monoaxial or polyaxial pedicle screw for fractured thoracolumbar vertebra can restore injured vertebral height. Compared with polyaxial pedicle screw, monoaxial pedicle screw endows stronger leverage which is more beneficial for restoring injured vertebral height and recovery of the damaged endplate in thoracolumbar short-segment posterior fixation.

Keywords: Clinical efficacy; leverage; monoaxial pedicle screws; polyaxial pedicle screws; thoracolumbar fracture

Submitted Jan 19, 2021. Accepted for publication Apr 08, 2021.

doi: $10.21037 /$ atm-21-881

View this article at: http://dx.doi.org/10.21037/atm-21-881

\section{Introduction}

Spinal fracture accounts for $14 \%$ of all bone fractures (1), with thoracolumbar fracture being the most common spinal injury $(2,3)$. Thoracolumbar fracture causes an unstable spine and leads to pain, disability, and even paraplegia (4). Posterior pedicle screw fixation is applied to maintain spinal stability and avoid further nerve damage (5). Some surgeons advocate that it is better to insert pedicle screws into the 
fractured vertebra because the reinforcement of fixation helps to improve kyphotic correction and biomechanical stability $(6,7)$. Other studies have shown that enhanced fracture-grade screws can improve spinal biomechanical stability (8-11). In addition, pedicle screw fixation combined with an intermediate screw in the fractured vertebra offers superior reduction and correction, fewer instrumental failures, and comparable clinical outcomes $(7,12,13)$.

Short-segment posterior fixation has been widely used for treating thoracolumbar burst fractures, which using pedicle screws with interconnected rods could restore the compressed vertebra and realign spinal kyphosis. Compared with long-segment posterior fixation, it not only limits the number of fused levels of spine, but also prevents excessive loads on adjacent discs. Moreover, short-segment stabilization has faster relief of pain, lesser tissue destruction than long segment with the same biomechanical stability (14).

However, there is no definitive guideline as to whether a monoaxial or polyaxial pedicle screw should be used for fractured vertebra in posterior fixation. The present study firstly compared the efficacy of using monoaxial pedicle screws versus polyaxial pedicle screws in short-segment posterior fixation for the treatment of thoracolumbar fractured vertebra. Specifically, we aimed to compare the leverage powers between polyaxial pedicle screw and monoaxial pedicle screw in restore of injured vertebral height in thoracolumbar fixation. We present the following article in accordance with the STROBE reporting checklist (available at http://dx.doi.org/10.21037/atm-21-881).

\section{Methods}

\section{Demographics}

From June 2012 to December 2018, 75 consecutive patients with thoracolumbar fracture who underwent shortsegment posterior fixation with a monoaxial pedicle screw (group A) or a polyaxial pedicle screw (group B) inserted in the fractured vertebra were retrospectively enrolled in this study. The inclusion criteria were: (I) short-segment posterior fixation including two levels with monoaxial or polyaxial pedicle screw via single compressed vertebra for thoracolumbar fracture; and (II) the thoracolumbar fracture had fused, and the pedicle screw had been removed at last follow-up. The exclusion criteria were: (I) patients with significant osteoporosis, endocrine diseases, vertebral tumour, tuberculosis, ankylosing spondylitis, or other diseases which may affect the vertebral structure; (II) patients with incomplete clinical records; and (III) a followup period of less than 18 months. This study has been approved by the Ethics Committee of The First Affiliated Hospital of Nanchang University. This study was designed in conformity with the Declaration of Helsinki (as revised in 2013). Individual consent for this retrospective analysis was waived.

\section{Clinical and radiographic records}

All patient clinical and radiographic data were recorded at admission, postoperative day 3 , and last follow-up (3 days after screw removal). Clinical records included thoracolumbar injury classification and severity scale (TLICS) scores (15), American Spinal Injury Association (ASIA) scores (16), Arbeitsgemeinschaft für Osteosynthesefragen (AO) Classification (17), hospital stay, injury-to-operation interval, and associated injury.

Radiographic data contained the prevertebral height ratio (the sum of the prevertebral heights of the superoinferior vertebrae divided by the two-fold height of the injured prevertebral height at lateral radiograph, Figure 1); the normal-to-injured vertebral height ratio (the sum median heights of the supero-inferior vertebrae divided by the two-fold height of the injured vertebral median at lateral radiograph, Figure 2); and the injured vertebral Cobb angle (the angulation between the upper endplate and the lower endplate of the injured vertebra, Figure 3). To address the potential sources of bias, all parameters were measured two times and taken the average values. The parameters were measured by two radiologists and a mean value was taken to decrease data bias.

\section{Statistical analysis}

All statistical analyses were performed by SPSS Statistics version 20 (IBM Co., Armonk, NY, USA). Data were presented as mean \pm standard deviation. Chi-square test was employed to reveal intergroup differences. Preoperative and postoperative data were compared by paired $t$-test. Independent samples $t$-test was used to compare corresponding parameters between the two groups. A probability less than 0.05 was considered statistically significant.

\section{Results}

\section{Outcome overview}

A total of 63 patients with an average age of $44.7 \pm 12.5$ years 


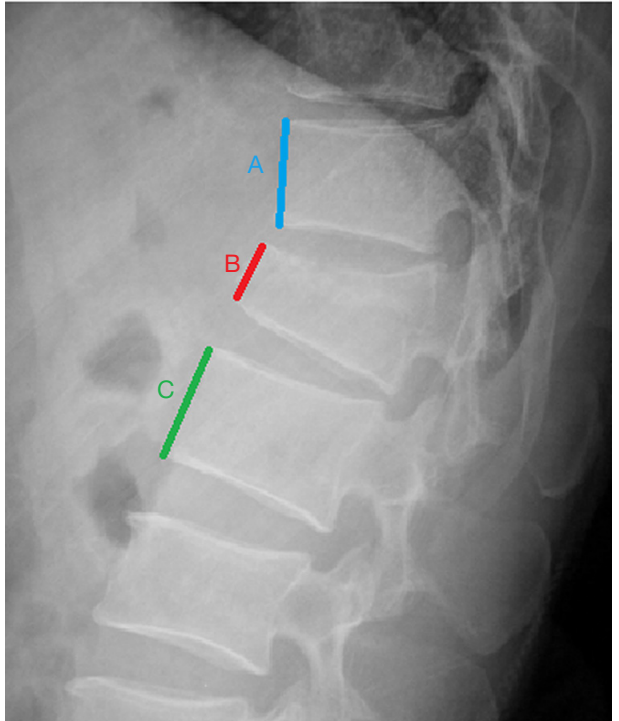

Figure 1 The prevertebral height ratio. This equals the sum of the prevertebral heights of the supero-inferior vertebrae (A and C) divided by the two-fold height of the injured prevertebral height (B) at lateral radiograph.

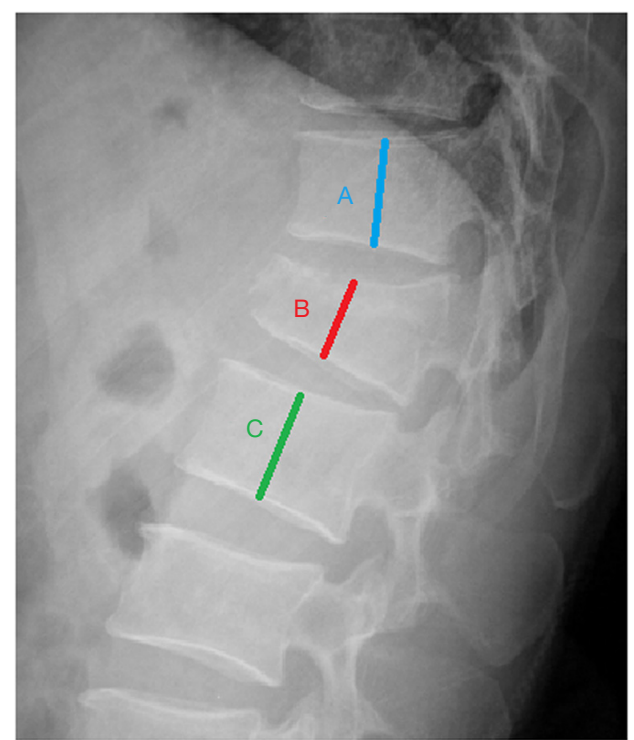

Figure 2 The normal-to-injured vertebral height ratio. This equals the sum median heights of the supero-inferior vertebrae (A and C) divided by the two-fold height of the injured vertebral median (B) at lateral radiograph.

were finally recruited, 12 patients with incomplete clinical records or follow-up were excluded. Of the 63 patients, 33 patients had treatment with monoaxial screws and were classified as group A (24 males and 9 females), and

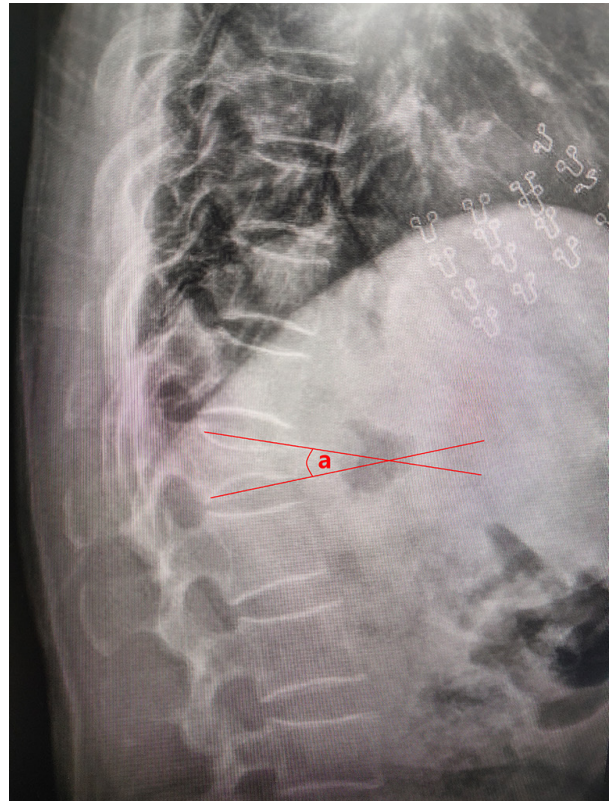

Figure 3 The Cobb angle of the injured vertebral.

30 patients had treatment with polyaxial screws and were classified as group B (18 males and 12 females). $\chi^{2}$ analysis revealed that there were no significant intergroup differences in age, gender, TLISS score, ASIA score, AO classification, and injury level between the two groups (all $\mathrm{P}>0.05$, Table 1). Moreover, there was no significant difference in hospital stay between groups $\mathrm{A}$ and $\mathrm{B}$ $(\mathrm{P}=0.267)$. However, injury-to-operation interval and associated injury were significantly different between the two groups $(\mathrm{P}=0.035, \mathrm{P}=0.025$, respectively), which could be due to the diversity in the degree of spinal injury and patient conditions.

\section{Questionnaires and AO classification}

As summarized in Table 1, the TLICS score for most patients was 5 or 6 points, and the ASIA scores were grades $\mathrm{D}$ and $\mathrm{E}$. The fractures were mainly located at L1 and L2. According to $\mathrm{AO}$ classification, the majority of vertebral fractures were types A1, A3, and B1 in both groups of patients.

\section{Radiographic findings}

Radiographic data verified that the prevertebral height ratio, the injured vertebral Cobb angle, and the normal-toinjured vertebral height ratio were significantly decreased 
Table 1 Patient demographics

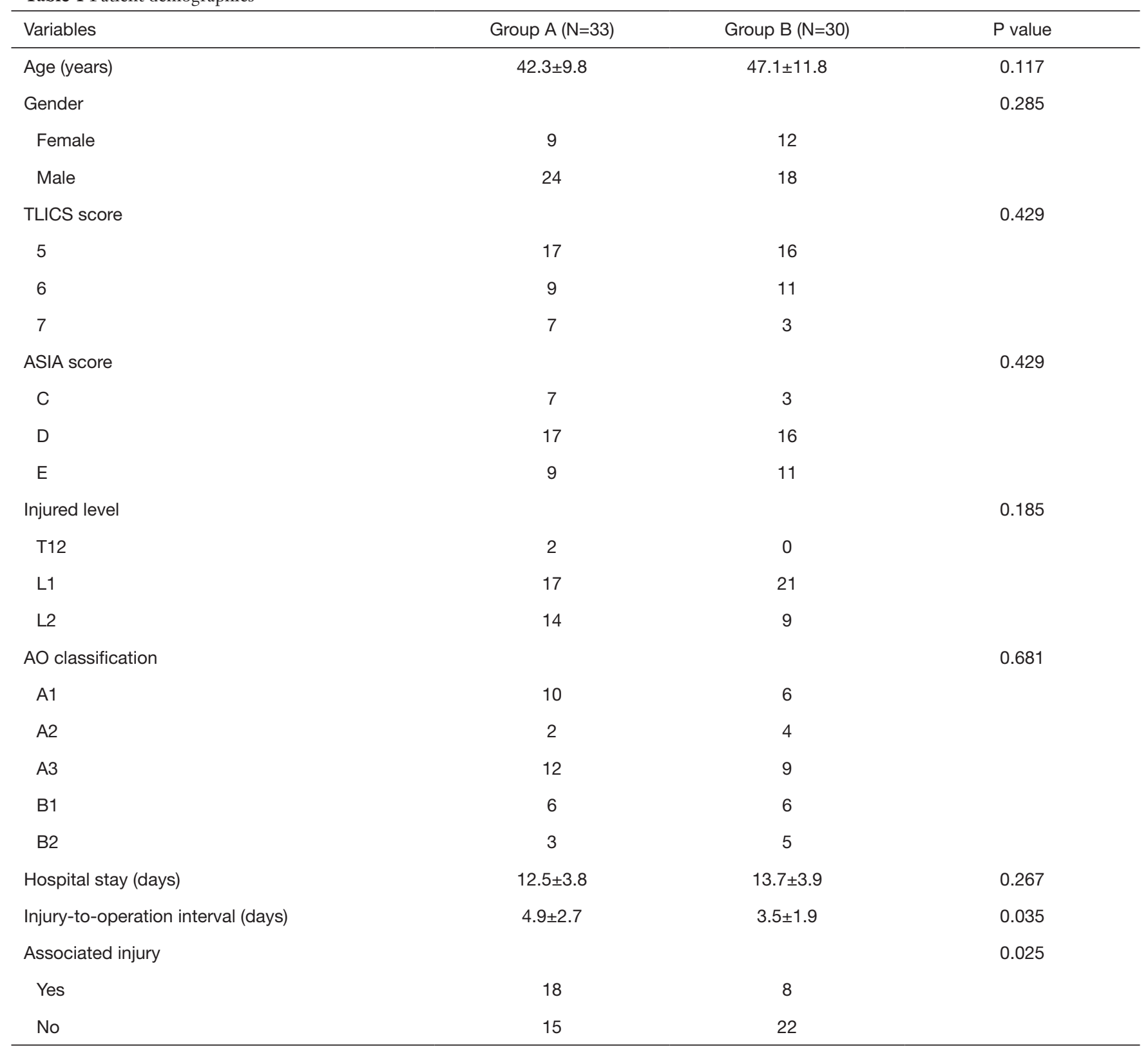

TLICS, thoracolumbar injury classification and severity scale; ASIA, American Spinal Injury Association; AO, Arbeitsgemeinschaft für Osteosynthesefragen; Group A, monoaxial screw group; Group B, polyaxial screw group.

between pre-operation and last follow-up for all patients (all $\mathrm{P}<0.05$; Table 2). However, the improvement in the prevertebral height ratio at last follow up was more marked in group A compared to group B $(\mathrm{P}=0.027)$. In addition, the normal-to-injured vertebral height ratio was ameliorated more distinctly at last follow-up in group A compared to group B $(\mathrm{P}=0.007)$.

\section{Discussion}

The benefits of short-segment posterior fixation for thoracolumbar fracture are well-accepted (6-13). Biomechanically, rods and pedicle screws can be used to thread the fractured vertebra and function as a "lordorizing" force that can restore vertebral height and correct spinal kyphosis (18). Compared with no screw in thoracolumbar 
Table 2 Radiographic data

\begin{tabular}{|c|c|c|c|}
\hline Variables & Group A (N=33) & Group B $(\mathrm{N}=30)$ & $P$ value \\
\hline \multicolumn{4}{|l|}{ Prevertebral height ratio (\%) } \\
\hline Pre-operation* & $1.5 \pm 0.4$ & $1.3 \pm 0.3$ & 0.047 \\
\hline Post-operation & $1.0 \pm 0.2$ & $1.0 \pm 0.3$ & 0.999 \\
\hline Last follow-up* & $1.0 \pm 0.1$ & $1.1 \pm 0.2$ & 0.027 \\
\hline \multicolumn{4}{|l|}{ Injured vertebral Cobb angle $\left(^{\circ}\right)$} \\
\hline Pre-operation & $19.0 \pm 10.2$ & $18.8 \pm 4.0$ & 0.926 \\
\hline Post-operation & $9.0 \pm 5.2$ & $8.4 \pm 2.1$ & 0.588 \\
\hline Last follow-up & $8.3 \pm 4.5$ & $8.7 \pm 2.2$ & 0.686 \\
\hline Pre-operation & $1.6 \pm 0.4$ & $1.5 \pm 0.3$ & 0.313 \\
\hline Post-operation* & $1.1 \pm 0.3$ & $1.4 \pm 0.2$ & 0.001 \\
\hline Last follow-up* & $1.1 \pm 0.3$ & $1.3 \pm 0.2$ & 0.007 \\
\hline Pre-operation vs. follow-up* & $\mathrm{P}<0.001$ & $P=0.007$ & \\
\hline
\end{tabular}

* indicates significant difference. Group A, monoaxial screw group; Group B, polyaxial screw group.

fractured vertebra, the use of monoaxial or polyaxial pedicle screws may achieve better kyphotic correction and vertebral height recovery $(7,8,10,12,19)$. However, to date, no literature has compared the efficacy of using monoaxial pedicle screws versus polyaxial pedicle screws in short-segment posterior fixation for the treatment of thoracolumbar fractured vertebra. The present study verified that both types of screws could restore injured vertebral height. Compared with the polyaxial pedicle screw, the monoaxial pedicle screw endows stronger leverage for thoracolumbar fractured vertebra.

In this study, the conditions of the patients recruited for the two groups were consistent as there were no significant differences in most of the demographic data including age, gender, TLICS score, ASIA score, injured level, and AO classification. Also, preoperative injured vertebral Cobb angle and normal-to-injured vertebral height ratio were similar between the two groups.

All radiographic outcomes had been ameliorated after surgery in both groups, which indicated that both monoaxial and polyaxial pedicle screws could restore injured vertebral height and realign spinal kyphosis. However, the prevertebral height ratio was improved more significantly in the monoaxial group at last follow-up compared to polyaxial group $(\mathrm{P}=0.027)$. The biomechanical properties of monoaxial pedicle screws give it stronger leverage, which can pry up the anterior portion of the injured vertebra since the junction between monoaxial screw head and shank/ thread is linear and fixed. Similarly, the normal-to-injured vertebral height ratio was decreased more significantly immediately post-operation and at last follow-up in the monoaxial group compared to the polyaxial group (both $\mathrm{P}<0.05)$. This suggested that the lever effect of monoaxial screws not only function at the spinal anterior column but also at the middle column (Figure 2), and this effect was greater than that observed with polyaxial screws.

Interestingly, although the prevertebral height ratio and the normal-to-injured vertebral height ratio were decreased more distinctly in the monoaxial group, the injured vertebral Cobb angles were similar between the polyaxial and monoaxial groups postoperatively. This may have been caused by the rods not being positioned straight enough for the monoaxial pedicle screw to exert sufficient lever effect on the lordotic angulation between the screws in the injured vertebra and the screws in the adjacent vertebrae.

Although polyaxial pedicle screws have been used widely, monoaxial pedicle screws are still relevant. In general, the polyaxial screw head is vulnerable to fatigue 

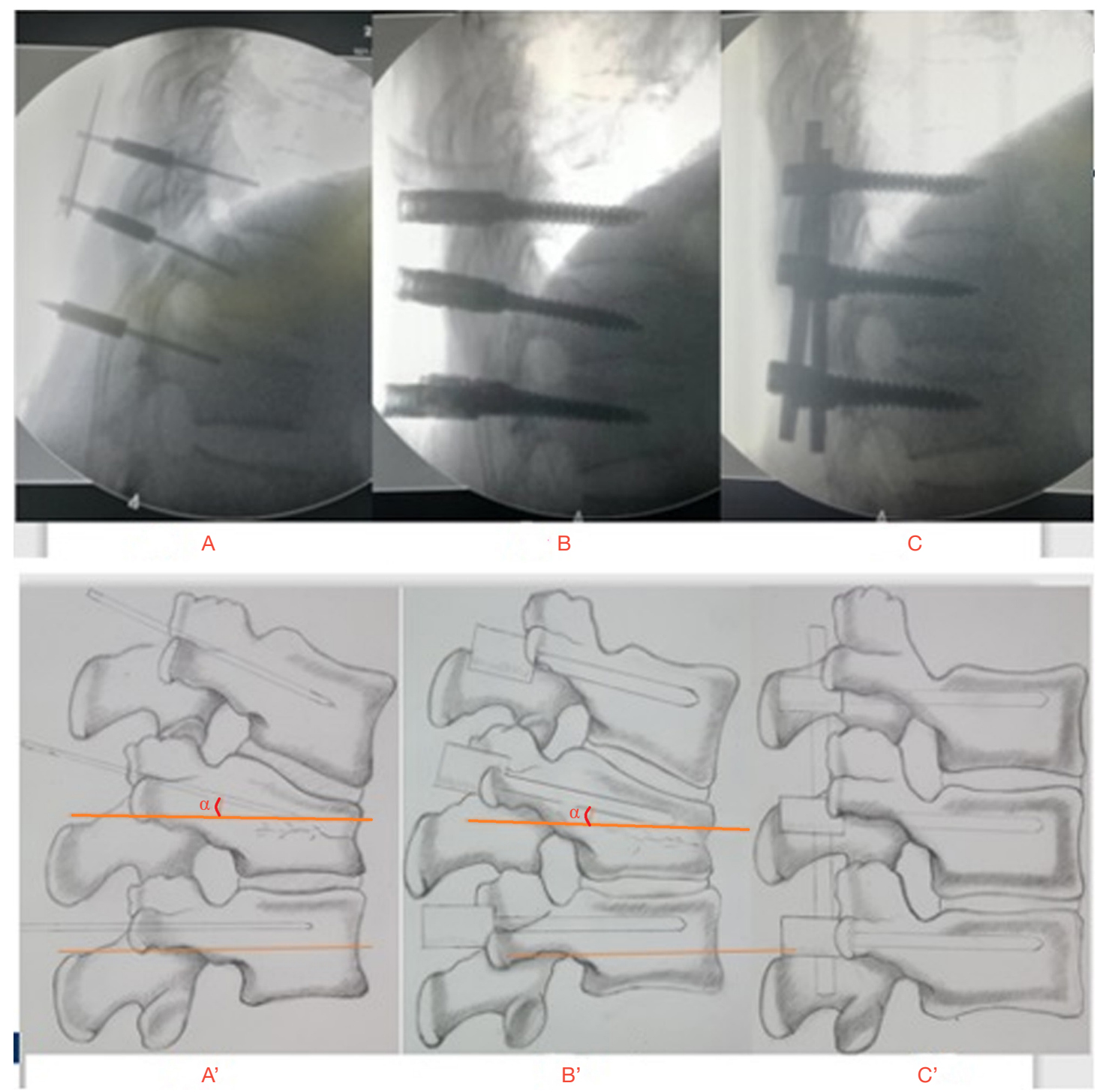

Figure 4 Our experience in the operation. Pedicle screws were inserted into the injured vertebra with an antero-inferior angle of 30 degrees to the cross section (A \& A'), and pedicle screws were placed at the supero-inferior vertebrae with an angle of 15 degrees to the cross section (B \& B'). The rods and nuts were then installed tightly. The screw shafts in the fractured vertebra would upwarp the compressed vertebra by paralleling the screw shafts in the adjacent vertebrae after fixing the screw-rod system (C \& C').

failure especially at the junction between the screw head and the shaft (20-22). In contrast, monoaxial screws with incorporated screw and shaft result in a stiffer construct and reduced von Mises stress on the screw (23). Compared with polyaxial pedicle screws, monoaxial pedicle screws inserted in the fractured vertebra show higher stability in flexion and extension of the spine (11). In addition, monoaxial screws show improved uplift and restoration of the collapsed upper endplate, thereby allowing for superior repair of the injured intervertebral disc. In our experience, the pedicle screws are inserted into the injured vertebra at an anteroinferior angle of 30 degrees to the cross section, and into the supero-inferior vertebrae with an angle of 15 degrees to the cross section (as shown in Figure 4). The rods and nuts are then installed tightly. Thus, the screw shafts in the fractured vertebra would upwarp the compressed vertebra by paralleling the screw shafts in the adjacent vertebrae after fixing the screw-rod system. Compared with polyaxial pedicle screws, monoaxial pedicle screws with greater lever effect played a more important role in this procedure. This 
may explain the significantly decreased prevertebral height ratio and normal-to-injured vertebral height ratio at last follow-up in the monoaxial group.

There were several limitations in this study. First, it was a retrospective study with a small sample. Second, patient parameters were collected from 6 years of clinical data. However, the materials used for surgical implants may not have been tightly controlled, and this may lead to clinical heterogeneity and data bias. Third, patientreported outcomes (PROs) were not documented and the association between PROs and radiographic parameters was not analyzed. Radiographic data may assist surgeons in optimizing surgical manipulations to improve quality of life. Therefore, a prospective study with more elaborate protocols will be beneficial for the development of improved techniques in thoracolumbar short-segment posterior fixation.

\section{Conclusions}

Both monoaxial and polyaxial pedicle screws when placed in thoracolumbar fractured vertebra for short-segment posterior fixation improve compressed vertebral height. However, compared with the polyaxial pedicle screw, the monoaxial pedicle screw offered stronger leverage which is more beneficial for restoring injured vertebral height and the recovery of the damaged endplate in thoracolumbar short-segment posterior fixation.

\section{Acknowledgments}

We thank the reviewers and editors for their helpful comments on this article.

Funding: This study was supported by the National Nature Science Fund of China (\#81860405).

\section{Footnote}

Reporting Checklist: The authors have completed the STROBE reporting checklist. Available at http://dx.doi. org/10.21037/atm-21-881

Data Sharing Statement: Available at http://dx.doi. org/10.21037/atm-21-881

Conflicts of Interest: All authors have completed the ICMJE uniform disclosure form (available at http://dx.doi. org/10.21037/atm-21-881). The authors have no conflicts of interest to declare.

Ethical Statement: The authors are accountable for all aspects of the work in ensuring that questions related to the accuracy or integrity of any part of the work are appropriately investigated and resolved. This study has been approved by the Ethics Committee of The First Affiliated Hospital of Nanchang University. This study was designed in conformity with the Declaration of Helsinki (as revised in 2013). Individual consent for this retrospective analysis was waived.

Open Access Statement: This is an Open Access article distributed in accordance with the Creative Commons Attribution-NonCommercial-NoDerivs 4.0 International License (CC BY-NC-ND 4.0), which permits the noncommercial replication and distribution of the article with the strict proviso that no changes or edits are made and the original work is properly cited (including links to both the formal publication through the relevant DOI and the license). See: https://creativecommons.org/licenses/by-nc-nd/4.0/.

\section{References}

1. Samelson EJ, Hannan MT, Zhang Y, et al. Incidence and risk factors for vertebral fracture in women and men: 25-year follow-up results from the population-based framingham study. J Bone Miner Res 2006;21:1207-14.

2. Tian Y, Zhu Y, Yin B, et al. Age- and gender-specific clinical characteristics of acute adult spine fractures in China. Int Orthop 2016;40:347-53.

3. Siebenga J, Leferink VJM, Segers MJM, et al. Treatment of traumatic thoracolumbar spine fractures: a multicenter prospective randomized study of operative versus nonsurgical treatment. Spine 2006;31:2881-90.

4. Denis F. The three-column spine and its significance in the classification of acute thoracolumbar spinal injuries. Spine 1983;8:817-31.

5. Sasso RC, Renkens K, Hanson D, et al. Unstable thoracolumbar burst fractures: anterior-only versus short-segment posterior fixation. J Spinal Disord Tech 2006;19:242-8.

6. Anekstein Y, Brosh T, Mirovsky Y. Intermediate screws in short segment pedicular fixation for thoracic and lumbar fractures: a biomechanical study. J Spinal Disord Tech 2007;20:72-7.

7. Mahar A, Kim C, Wedemeyer M, et al. Short-segment fixation of lumbar burst fractures using pedicle fixation at 


\section{Page 8 of 8}

the level of the fracture. Spine 2007;32:1503-7.

8. Baaj AA, Reyes PM, Yaqoobi AS, et al. Biomechanical advantage of the index-level pedicle screw in unstable thoracolumbar junction fractures. J Neurosurg 2011;SPI 14:192-7.

9. Bolesta MJ, Caron T, Chinthakunta SR, et al. Pedicle screw instrumentation of thoracolumbar burst fractures: biomechanical evaluation of screw configuration with pedicle screws at the level of the fracture. Int J Spine Surg 2012;6:200-5.

10. Norton RP, Milne EL, Kaimrajh DN, et al. Biomechanical analysis of four- versus six-screw constructs for shortsegment pedicle screw and rod instrumentation of unstable thoracolumbar fractures. Spine J 2014;14:1734-9.

11. Wang H, Li C, Liu T, et al. Biomechanical efficacy of monoaxial or polyaxial pedicle screw and additional screw insertion at the level of fracture, in lumbar burst fracture: An experimental study. Indian J Orthop 2012;46:395-401.

12. Farrokhi MR, Razmkon A, Maghami Z, et al. Inclusion of the fracture level in short segment fixation of thoracolumbar fractures. Eur Spine J 2010;19:1651-6.

13. Gelb D, Ludwig S, Karp JE, et al. Successful treatment of thoracolumbar fractures with short-segment pedicle instrumentation. J Spinal Disord Tech 2010;23:293- 301.

14. Sallam AM, Abdel Ghany WA, Ali AK, et al. Shortsegment posterior fixation with index level screws versus long-segment posterior fixation for thoracolumbar spine fracture: angle of correction and pain. Egypt J Neurosurg 2018. https://doi.org/10.1186/s41984-018-0012-9

15. Vaccaro AR, Lehman RA Jr, Hurlbert RJ, et al. A new classification of thoracolumbar injuries: the importance of injury morphology, the integrity of the posterior ligamentous complex, and neurologic status. Spine

Cite this article as: Yao W, Zhou T, Huang K, Dai M, Mo F, Xu J, Cao Z, Lai Q, Xie B, Guo R, Zhang B. A comparison of monoaxial pedicle screw versus polyaxial pedicle screw in shortsegment posterior fixation for the treatment of thoracolumbar fractured vertebra. Ann Transl Med 2021;9(8):669. doi: 10.21037/atm-21-881
Yao et al. Monoaxial pedicle screw for thoracolumbar fracture

2005;30:2325-33.

16. Marino RJ, Graves DE. Metric properties of the ASIA motor score: subscales improve correlation with functional activities. Arch Phys Med Rehabil 2004;85:1804-10.

17. Carlo B, Schroeder GD, Kepler CK, et al. The AOSpine sacral fracture classification. Global Spine J 2016;6:686-94.

18. Ren HL, Wang JX, Jiang JM. Is short same-segment fixation really better than short-segment posterior fixation in the treatment of thoracolumbar fractures? Spine 2018;43:1470-8.

19. Park SR, Na HY, Kim JM, et al. More than 5-year followup results of two-level and three-level posterior fixations of thoracolumbar burst fractures with load-sharing scores of seven and eight points. Clin Orthop Surg 2016;8:71-7.

20. Kubosch D, Kubosch EJ, Gueorguiev B, et al. Biomechanical investigation of a minimally invasive posterior spine stabilization system in comparison to the Universal Spinal System (USS). BMC Musculoskelet Disord 2016;17:134.

21. Fogel GR, Reitman CA, Liu W, et al. Physical characteristics of polyaxial-headed pedicle screws and biomechanical comparison of load with their failure. Spine 2003;28:470-3.

22. Stanford RE, Loefler AH, Stanford PM, et al. Multiaxial pedicle screw designs: static and dynamic mechanical testing. Spine 2004;29:367-75.

23. Wang H, Zhao Y, Mo Z, et al. Comparison of shortsegment monoaxial and polyaxial pedicle screw fixation combined with intermediate screws in traumatic thoracolumbar fractures: a finite element study and clinical radiographic review. Clinics 2017;72:609-17.

(English Language Editor: J. Teoh) 